Volume 8, No.6, November -December 2019

International Journal of Science and Applied Information Technology

Available Online at http://www.warse.org/ijsait/static/pdf/file/ijsait02862019.pdf

https://doi.org/10.30534/ijsait/2019/02862019

\title{
Technique of Image Compression: A Review
}

\author{
Maali Alslehat ${ }^{1}$ AKram Aldulaimi ${ }^{1}$ Mohammed Otiar $^{1}$ \\ Department Of Computer Science, Amman Arab University, Amman, Jordan \\ maali.fathi@yahoo.com
}

\begin{abstract}
An image compression technique eliminates unrelated information or/and redundant, and resource for encode leftover. Almost, it is repeatedly essential to tossing a way both relevant information and not redundant information to attain the compression that essential. By the way, the ploy is discovered approaches that permit significant info to be resourcefully extracting and representing. As we look to the future, the need to transmit images and store will only continue to increase faster than the available capability to process all the data. Multimedia data whether audio, graphics and video data which is uncompressing requires considerable transmission bandwidth and storage capacity. So, this leads to the need techniques of compression of images and all applications of multimedia to save time of transmission and storage. The purpose of this paper is to show different techniques that used for compressing images in two sides lossy compression and lossless compression. Also, we have shown in this research paper the motivation behind using these techniques and the benefits of compression techniques.
\end{abstract}

Key words : Image Compression Techniques, Lossless Technique, Lossy Technique, A Review paper for image compression.

\section{INTRODUCTION}

Image is defined as a two dimensional functions, e.g. let $\mathrm{g}(\mathrm{x}, \mathrm{y})$ is a function and it's depending on two variables so $\mathrm{g}$ is dependent on independent variables $\mathrm{x} \& \mathrm{y}$, in which $\mathrm{y}$ and $\mathrm{x}$ are coordinates of plane. We distinguish when we are taking the image, which is function of $x / y$, then with helping of $x / y$ we can calculating the level of intensity for image or can saying pixel. As we are knowing pixel value lies between ( 0 255) [1]. Techniques of image compression play role that very important in many applications. It is helping in reducing the bytes number of an original image exclusive of any misrepresentation in its quality, so that it will take fewer time, hard space of disk or transmission bandwidth to sending an info from one place to another for example, Z 24 bit image with 512x512 intensity will occupy 768 (Kilo byte) on a storage disk, and a paired of image of this size won't fit in a only disk of floppy. So, to transfer an image over a 28.8 kilo bytes modem will take near four minutes. The motivation behind techniques of image compressing is to decrease the total data require for represent sample digital image and then decrease costing of transmission and storage [2].The image compression in general is to get rid of the duplicates in as accurate a picture as possible.

Decompression is the reverse process of pressure In this paper, we explained the techniques of lossless compression (LZW, RLW, Huffman and arithmetic encoding )and techniques of compression lossless(DCT , sub band coding ,BTC and vector quantization). We mentioned some of the bases that evaluate the techniques of image compression, and then some of the benefits of compression were listed. In the last part of the paper was compared through table , Lossless technique and lossy technique, the comparison included the algorithms used, their uses and the images to which these algorithms apply, and we mentioned the pros and cons of each

By Jau Ji Shen et al presented, the vector quantization is basing on technique of image compression. In this method encoding of the variance maps between the images that original and image that compressed is adjusting and after that it is restoring in VQ compressing version. Results of this experiments show that although this scheme need to provide extra data, it can enhance the Vector quality of quantized compressed image, in addition, be adjusted according to the variance maps from technique of lossy compression to compression of lossless.[3]

In 2011, presented the approach of the technique of image compression lossless using the novel concept of image folding [4].This method is proposed uses the properties of adjacent neighbor redundant of prediction. In this technique, column fold is followed by row fold is applying iterative on the images till the size of image in order to reduce to a smaller predefined value. This technique is then comparing with the existing image compression lossless algorithms and is obtained results show a performance of comparative of different approaches. Folding of data technique is a simple technique for compressing images which offers better performance and offer lesser computation complex as comparing to the SPIHT method of lossless compression. 
Maali Alslehat et al., International Journal of Science and Advanced Information Technology, 8 (6), November - December 2019,30 - 35

Firas et al presented a novel technique for compressing the images namely as five module method FMM. In this technique they transform each pixel value in $8 \times 8$ block in to a multiple of five for every of RGB array [5]. Then After that, the value is dividing by five to obtaining new value that is known as bit length for every intensity and used fewer space of storage than the values that original which is eight bits. The advantage of this technique is providing high PSNR durig it is low compression ratio. This technique is good for bi-level such as white and black for medical image where the pixels of the image is presenting uses 1 byte.

There are image compression method which is using dissimilar embedded wavelet bases on coding of image with Huffman encoding for additional compression. In this method, they implemented the SPIHT and EZW and algorithms with huffman encoder[6], which is used dissimilar of wavelet for compressing and after that comparing between the PSNR and rates of bit for these families are created. These methods were executed on different images, and it is given the results have good quality and it also gives maximal ratio of compression as comparing with the previous existing lossless image compression methods [7].

In 2015, by Mohammed Otair and Alkhalayleh are presented technique based on Huffman tree by decomposing the tree in to four groups and named this technique is LM-DH technique. This technique works in which first stage in LM-DH technique is reading the gray scale of original image by the MATLAB, then calculate the frequency for every gray level in the input picture, and gather then in matrix (M), then is sorted $\mathrm{M}$ in descending order built on frequency of gray level. Compressing the image by using the technique of huffman to discover size of the classical huffman compressed image, in order to comparing it later with the size image of LM-DH [8].

Also, by Mohammed Otair and Alshami are presented lossless preprocessing method that improves the lossy methods through making a minimize rates of distortion in the compression. The idea of core for this method (MDT) depending.

\subsection{The main Problem}

The main problem It is bytes number of an original image, so that it will take over time, and need amount of space larger to transmission an info from one place to another.

\subsection{Benefits image compression}

- Space of storage compressing data file allows one to store more files in the space of storage that is available[13].

- Transfer speed compressed and bandwidth files contain fewer bits of information than uncompressed files, and as a consequence, using a smaller amount of bandwidth when we download them [13].

- The cost of storing information are reducing by compressing the files for storage because more files can be stored in available space of storage when it is compressed[19].

- Accuracy is reduced the chance of errors of transmission since less bits are transferring [12].

- Security is providing a level of security against monitoring of illegitimate [12].

\section{IMAGE COMPRESSION TECHNIQUES}

Through the two decades last, a rang for techniques of compression are advanced to address main challenges are facing by digital image processing. These techniques of compression can be categorized generally as technique of lossless compression and technique of lossy compression [10].

\subsection{Techniques of Lossless compression}

Techniques of Lossless compressors "Fig. 1" have two steps of algorithms. The first step is transforming the actual image to some other formats in which the redundancy of inter pixel is decreased. Then last step is using entropy encoder in order to remove the redundant code. The lossless decompression is a model converse procedure of the lossless compression [11].
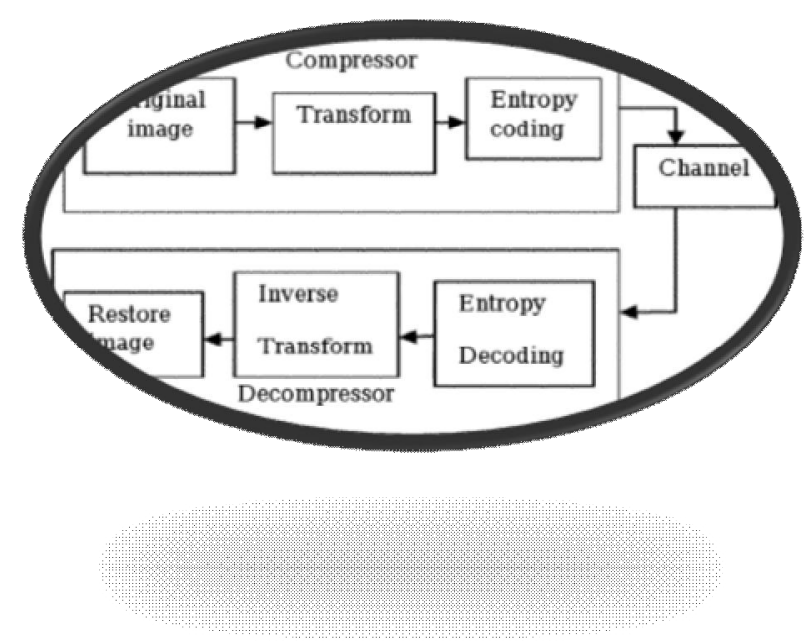

Figure 1: Image compression by lossless techniques 
Maali Alslehat et al., International Journal of Science and Advanced Information Technology, 8 (6), November - December 2019,30 - 35

\subsubsection{Run Length Encoding}

It is techniques for compressing the image. it is used for serial information only. This method is replacing series of same pixels, with the codes that shorter [2]. It is representing as $\{\mathrm{v}, \mathrm{r}\}$ in which $\mathrm{v}$ is representing intensity of pixel and $\mathrm{r}$ is representing the intensity of the number pixels in original code.

Example : 90, 90, 90, 66, 66, 66, 66, 66, 88

$\{90,3\},\{66,5\},\{88,1\}$.

\subsubsection{Huffman encoding}

This technique was established by huffman. This methodology is using to eliminate redundant code in the image. This technique, we are coding the symbols by the occurrence of statistical for frequencies and accordingly we generate a tree. By this technique, codes with the utmost frequency will result in code that shorter symbols, words, and with lesser frequency will have long code words[2]. It is also called variable length coding

\subsubsection{LZW Encoding}

Limpel-Ziv-Welch is a method used to compress data. Lempel, Ziv, and Welch established it. Technique LZW is encoding functioning based on the amount of multiplicity of bits series in the pixel to be encoded. This is an error free approach for compressing is focusing on remove redundancy of spatial. It is assigning code words that length is fixed to variable length arrangements of sources symbols. It plays on file formats such as, GIF, TIFF, and PD [2].

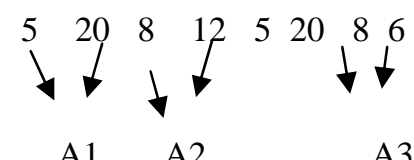

data is compressing: $\mathrm{A} 1 \mathrm{~A} 2 \mathrm{~A} 1 \mathrm{~A} 3$

\subsubsection{Arithmetic Encoding}

This approach was produced by rissanen in which the fart symbol is decoded and encoded last[12]. Arithmetic encoding is built on following principles. The symbols that alphabet must not infinite. All possible symbols series of give length must not infinite. The number of actual number in the interval $[0,1]$ can allocate a unique subinterval for any given input symbols sequence[13].

\section{2 Lossy compression Techniques}

Is the removal of all the repeated data, the advantage of this technique is that the compression ratio is high and is the most used, but there is a loss of data which means [14] that the compressed image does not match the images entered completely. The"Fig.2"shows how technique lossy compression technique.

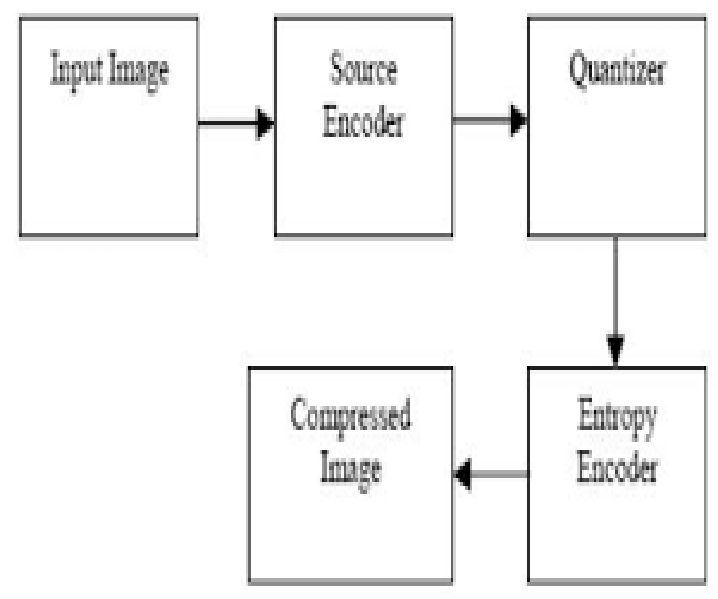

Figure 2: Image compression by lossy techniques

\subsubsection{The discrete cosine transform DCT}

DCT could be a technique lossy compression that is wide employed in audio compression and space of image. DCT are accustomed transform information within the series summation of trigonometric function waves oscillatory at completely dissimilar frequencies. There resemblance with DFT (Discrete Fourier transforms) however DCT involves uses of cosine circular function is rather more active as less function is required to approximate a signal [15]. This method is used in one-dimensional and two-dimensional DCT.

\subsubsection{The sub band coding}

The coding of sub band split bands of frequency if a signal and then every sub from band is coding by encoder. A decoder is decoding the sub of signal band, then it samples and passes by synthesis filters. SBC in general using in coding of speech and coding of image[16].

\subsubsection{Block Truncation Coding (BTC)}

The encoding truncation encoding is a technique for compressing images. BTC divide the input image into small $n$ $\mathrm{x} n$ pixel sub-blocks, then after dividing the input image, The gray level reduction is performed by the quantizer. This reduces the quantity of gray levels within each pixel [16].

\subsubsection{Vector quantization}

A vector quantization is divided into two processes. The primary is encoding the code of word, and the second is decoder. The encoder takings vector as an input and outputs the index of the code word with lowermost distortion that is calculating by evaluate the Euclidian distance between the input vector and every code word within the codebook. When a nearest code word is found, the index of that code word is distributed over a channel. Once the encoder receives the index of the code word, It is replacing the index with the 
Maali Alslehat et al., International Journal of Science and Advanced Information Technology, 8 (6), November - December 2019,30 - 35

associated code word [17]. The "Fig.3"shows how work the technique.

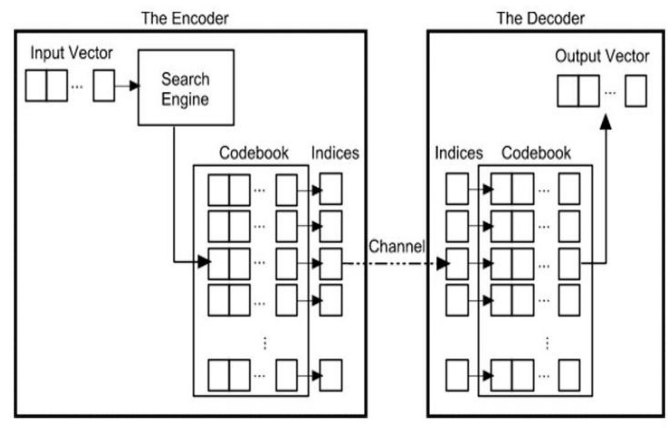

Figure 3 : Vector quantization

\section{EVALUATION OF IMAGE COMPRESSION}

To compute the image quality there are dissimilar parameters, usually used are peak signal to noise ratio and mean squared error. In this section, different parameters are discussed. The MSE is the cumulative squared error among the actual and compressed picture is computed by [18]:

Equ 1 :

$$
\text { MSE }=\frac{1}{\operatorname{MN}} \sum_{i=1}^{M} \sum_{j=1}^{N}(x(i, j)-y(i, j))^{2}
$$

PSNR is an amount of the peak signal-to-noise ratio between the real and image compressing is computed by:

Equ 2:

$$
\operatorname{PSNR}(d B)=10 \log _{10}\left(\begin{array}{c}
255^{2} \\
\text { MSE }
\end{array}\right)
$$

Where $\mathrm{i}(\mathrm{x}, \mathrm{y})$ is the actual image, $\mathrm{z}(\mathrm{x}, \mathrm{y})$ is the version of approximated (really image decompressing) and N,M are the dimensions of image. An lesser value for MSE means lesser error, and as seen from the inverse relation between the PSNR and MSE, this is translating to a soaring PSNR value.

\section{CONCLUSION}

\subsection{Conclusion}

In this paper reviewed, Image compression techniques lossy image compression technique is most commonly used because it gives high compression ratio. Technique of Lossless compression is used when we want image that original and reconstructed image to be identical. The objective of compression and how each technique was worked out was clarified. Also, we offer comparison between compression techniques based on its properties in order to be the idea is clear and comprehensive to the reader or researcher.

\subsection{Suggestion}

The domain will continue to researchers interesting at now to come and try to find a compression method without loss of data with a very high compression ratio. 
Maali Alslehat et al., International Journal of Science and Advanced Information Technology, 8 (6), November - December 2019,30 - 35

\begin{tabular}{|c|c|c|}
\hline FACTORS & $\begin{array}{l}\text { LOSSY } \\
\text { COMPRESSION }\end{array}$ & LOSSLESS COMPRESSION \\
\hline Definition & $\begin{array}{l}\text { Lossy compression is the class for } \\
\text { encoding data techniques that are using } \\
\text { approximations that inexact for } \\
\text { representing the content. These } \\
\text { methods are using to decrease the size } \\
\text { of data for handling, storage, and } \\
\text { transmit content. }\end{array}$ & $\begin{array}{l}\text { Lossless compression is the class for } \\
\text { compressing data algorithms that are } \\
\text { allowing the original data to be perfect } \\
\text { reconstruct from the data is compressed. }\end{array}$ \\
\hline Algorithms & $\begin{array}{l}\text { DCT, Transform coding, } \\
\text { Dectangle segmentation and } \\
\text { sparse } \\
\text { matrix storage (RSSMS). }\end{array}$ & $\begin{array}{l}\text { LZW, RLW, Huffman, Arithmetic } \\
\text { encoding, and Shannon fano coding. }\end{array}$ \\
\hline Uses & Audio, video and image. & Sound, images and programs or texts. \\
\hline Images & $\begin{array}{l}\text { GUI and JEPG are formats of lossy } \\
\text { images. }\end{array}$ & $\begin{array}{l}\text { BMP, PNG and RAW are formats of } \\
\text { lossless. }\end{array}$ \\
\hline Video & $\begin{array}{l}\text { Formats that Common like H-264, } \\
\text { WMV and MKV are lossy. H- } 264 \text { can } \\
\text { give lesser files with upper qualities } \\
\text { than previous generation for coding } \\
\text { video because it has a lesser procedure } \\
\text { that is better to choose data for } \\
\text { throwing out. }\end{array}$ & $\begin{array}{l}\text { Few lossless formats of video are } \\
\text { common consumer use, formats would } \\
\text { results in files of video take up amount } \\
\text { that huge of space. }\end{array}$ \\
\hline Audio & $\begin{array}{l}\text { OOG, mp4 and } \mathrm{mp} 3 \text { are audio formats } \\
\text { of loosy. }\end{array}$ & $\begin{array}{l}\text { FLAC, WAV and ALAC are formats of } \\
\text { lossless. }\end{array}$ \\
\hline Advantages & $\begin{array}{l}\text { Multimedia files are could much lesser } \\
\text { than its actual size. It can decrease } \\
\text { sizes of file much more than } \\
\text { compression of lossless. }\end{array}$ & $\begin{array}{l}\text { It is maintaining quality. } \\
\text { Transformation to other formats } \\
\text { possible without losing in information of } \\
\text { audio. }\end{array}$ \\
\hline Disadvantages & $\begin{array}{l}\text { Transformation to another formats } \\
\text { only with losing in information of } \\
\text { audio. It can not use in every type of } \\
\text { files because it is working by remove } \\
\text { data. Data and text can not be } \\
\text { compressing they have not redundant } \\
\text { information. }\end{array}$ & $\begin{array}{l}\text { It does not decrease the size of file as } \\
\text { much as lossy compressing. Technique } \\
\text { of lossless encoding cannot accomplish } \\
\text { compression for high levels. }\end{array}$ \\
\hline $\begin{array}{l}\text { Characteristics } \\
\mathbf{z}\end{array}$ & $\begin{array}{l}\text { Ratio of compression is big and good for graphic } \\
\text { image. }\end{array}$ & $\begin{array}{l}\text { Documents are scanning and imaging formats. } \\
\text { Flexible RLE, LZW and CCITT. }\end{array}$ \\
\hline
\end{tabular}


Maali Alslehat et al., International Journal of Science and Advanced Information Technology, 8 (6), November - December 2019,30 - 35

\section{REFERENCES}

1. Madhu and Dalal. (2017). Review Paper on Image Compression Using Lossless and Lossy Technique. International Journal of Advance Research, Ideas and Innovations in Technology, 3(2), pages 873-878.

2. Ijmulwar and Kapgate. (2014). A Review on - Lossless Image Compression Techniques and Algorithms. International Journal of Computing and Technology, $1(9)$, pages 457-460.

3. Jau-Ji Shen and Hsiu-Chuan H. (2010) An Adaptive Image Compression Method Based on Vector Quantization, IEEE, pages 377-381.

4. Mohamed and Kingston, F. Image compression using VQBTC. IEEE Transactions on Communications.

5. Suresh Y., Smita N. and Krishnan K. (2011). Lossless Image Compression based on Data Folding, IEEE, pages 999-1004.

6. Akhand P. S., Potnis, and Kumar. (2016). A REVIEW ON LATEST TECHNIQUES OF IMAGE COMPRESSION. International Research Journal of Engineering and Technology (IRJET), 3(7), July

7. Ragab, Abdalla and Mohmed, M.S. H. (1998). Efficiency of Analytical Transforms for Image Compression. $15^{\text {th }}$ National Radio Science Conference, pages 24-26, , Cairo- Egypt.

https://doi.org/10.1109/NRSC.1998.711461

8. Otair and Mahmoud A. (2015). A New Lossless Method of Image Compression by Decomposing the Tree of Huffman Technique. International Journal of Imaging and Robotics, 15(2).

9. Otair and Ahmed A. (2018). Enhancing Quality of Lossy Compressed Images using Minimum Decreasing Technique. International Journal of Advanced Computer Science and Applications (IJACSA), 9(3). https://doi.org/10.14569/IJACSA.2018.090353

10. Kaur and Choudhary, P. (2016). A Review of Image Compression Techniques. International Journal of computer Application, 7(1), pages 8-11. https://doi.org/10.5120/ijca2016909658

11. Kaur and Kaur. (2013). A Survey of Lossless and Lossy Image Compression Techniques. International Journal of Advanced Research in Computer Science and Software Engineering, 3(2), February.

12. Fisher Y. (1994). Fractal Image Compression: Theory and Applications. Springer-Verlag.

13. Goyal, DR-N. Gupta, and Dr. P. Gupta, G. (2017). A REVIEW PAPER ON COLOR IMAGE COMPRESSION TECHNIQUES. International Journal of Advance Engineering and Research Development, 4(8), August.

14. Abualigah, L. M. Q. (2019). Feature selection and enhanced krill herd algorithm for text document clustering. Berlin: Springer.

https://doi.org/10.1007/978-3-030-10674-4

15. P. Kavitha. (2016). A Survey on Lossless and Lossy Data Compression Methods. International Journal of
Computer Science \& Engineering Technology (IJCSET), 7(3), pages 2229-3345. March.

16. A. Singh, A. Potnis, and Kumar A. (2016). A Review on Latest techniques of Image Compression. International Research Journal of Engineering and Technology (IRJET), 3(7), pages 2395 -0056, July.

17. Sachin D. (2011). A Review of Image Compression and Comparison of its Algorithms. In IJECT, 2(1), March.

18. Dr. B. E. Reddy and N. (2012). A Lossless Image Compression Using Traditional And Lifting Based Wavelets. Signal \& Image Processing: An International Journal (SIPIJ), 3(2), April. https://doi.org/10.5121/sipij.2012.3216

19. Nirbhay K., and Narayan S. (2013). Review of Image Compression and Comparison of its Algorithms. In International Journal of Application or Innovation in Engineering \& Management (IJAIEM), 2(12), pages 2319 - 4847, December. 